\title{
Research on Translation Characteristics Based on English Corpus Yang Lin
}

Inner Mongolia University, Foreign Language School, Hohhot, Inner Mongolia, China, 010021

\author{
Keywords: Translation, Characteristics, English Corpus
}

\begin{abstract}
Corpus Translation Studies has become an important branch of Translation Studies. Over the past twenty years, translation theory has gone through two turns, one is the translation studies have jumped out of the limitations of the faithful and focused on the translation of the text, with particular attention to the regularity description of translation behavior. The Corpus and Corpus Linguistics technology is a powerful tool that can explain the essential characteristics of language translation and can provide evidence for the translation process itself. Therefore, Translation Studies based on English Corpus has become the focus of translation studies and this paper focuses on feature of translation based on English Corpus.
\end{abstract}

\section{Introduction}

Translation feature also known as universal translation is "different from the source language translation of this presented some typical, cross-language, there are certain universal features." Some of our scholars to translate the characteristics of the period and divided before Corpus Corpus-based period for the Baker watershed article published in 1993 in Corpus Linguistics and Translation Studies: Implications and Applications. Baker first proposed the concept of the text translation feature on the assumption that "the typical characteristics of language translation of the text rather than the source text embodied, and as a result of these features are not language-specific system of interaction."

The assumptions include six areas: (1) manifest translation degree significantly increased; (2) disambiguation and simplified; (3) grammatical properties; (4) avoid duplication; (5) over the reproduction target language features; (6) translation process often leads to some languages characterized by a particular type of distribution. Characteristics of Translation Studies Corpus-based Translation Studies has since become a hot research, most of these studies focus on the manifestation, simplification and normalization translation and other features to expand, both confirmed that there are falsified.

Manifest, also known as clarity, it was first used by Israeli scholars Blum-Kulka put forward. She believes that translators tend to add some cohesion markers and additional information to help the reader understand the translation, so the translations are often verbose than the original. Baker also believes that translation has to express the original trend information may be implicit, is obvious trend. Translation language study, the concept of manifest and simplification are closely related. Compared with the source language and the target language mother tongue text, translated text in vocabulary, grammar, syntax and style and other aspects are more streamlined. The standardization of the language refers to the translation with the "comply or even exaggerate the typical characteristics of the target language and expressions of the trend."

At present, some foreign studies confirmed the presence of the translation feature, while others questioned the translation feature. However, these studies basically reached a consensus that "Translation is a special text is different from the source text, but also translated into the languages of the different." In recent years, domestic Corpus Translation Studies has developed rapidly, the study to explore "whether on the basis of language translation feature from close observation of language translation over the English translation on the results can be generalized to 'translate feature' or 'Universal Translator'" "in English and Chinese translation involved such a large span, the Chinese translation of whether exhibits the same characteristics." 


\section{The Translation Studies Based on English Corpus}

Since the early 1990s, with the rapid development of corpus linguistics and the description of the continuing impact of Translation Studies, Mona Baker, led to a number of research scholars corpus linguistics applied to translation studies, Jian create a new translation Corpus translation studies a corpus-based translation studies. One advantage of this new paradigm is to learn from corpus linguistics research methods and results, focusing on empirical research, to obtain the translation data from the corpus, and then for statistical analysis, the results are more objective and scientific research, which has traditional translation studies do not have the advantage. Baker first proposed corpus-based translation studies. $\mathrm{Hu}$ Bao the corpus translation studies is defined as: "In the corpus-based, real bilingual corpus or translation corpus for the study to statistical data and theoretical analysis methods, based on linguistics, literature and cultural theory and translation theory research, system analysis essence of translation, the translation process and the translation of content such phenomena. "

Translated text is often translated researchers call "Translated", often with defined color jump that it has many different characteristics .Duff standard deviation from the native language referred to this kind of language translation "third language", because it affects primitive that sounds like a foreign language to translate. Frawley language called "third code", Frawley is "the first to translate the language as the existence of independent linguists ".

In recent years, domestic Corpus Translation Studies research has achieved fruitful results. Xiao Zhonghua, Dai Guangrong also believes that since the 1990s, Corpus - based Translation Studies has made considerable progress to descriptive translation learn tremendous vitality. Non-Wang Ke argues, Corpus Translation Studies (corpus-based translation studies) way after ten years of theoretical explanations and empirical research has developed into a new paradigm of research, research involving the common features of language translation, the translator Style translation conversion process, of which the translation of the most prominent features of the study.

But the study results are mainly foreign translation feature comparison between English and other European languages generated based on whether these findings apply to the translation feature in Translation requires further observation and testing. Characteristics of Translation Studies scholars focused on introducing foreign translation and characteristics of the Translation Process Explicitation empirical research.

\section{The Characteristics of Translation}

Translation feature is also known as Universal Translator, the classic definition from Baker: "Typical language translation features appear in the text, with different linguistic features primitive, and these features are not typical language-specific language-system interference product. "a typical linguistic features such language translation by comparing the translated text and original text (such as translation Chinese and native Chinese) analysis, which can provide a theoretical basis for the translation practice, and help build and translation teaching effect of translation theory improved. Translation feature hypothesis is that these typical features language translation from the translation process itself, rather than from the effects of two language system differences. Huang Libo, Wang Ke translated for non-universality is defined as. "Translation language as objective language variants, with the native language from the overall performance out of some of the regular features of the language source language or target language," Wang Ke Fei, Huang Libo to Baker for the sector, to translate research into features: front corpus (pre-corpus) based on the period and the corpus (corpus-based) period. Halverson pointed out the characteristics of translation problems: study focused on descriptive and inductive research, the lack of explanatory research. And for about the translation feature hypothetical critical point, Toury points out that research is not a translation feature or not there is a problem, but the explanatory power problem, namely how to use a variety of tools to translate the concept of the phenomenon better explanation.

In recent years, research-based translation feature Corpus becomes the focus of the research corpus linguistics, mainly in the explicitation / implicitation, simplification and normalization. Our 
research is mainly related to explicit and implicit oriented, so then reviewed the major domestic and foreign theoretical perspectives and research on explicit and implicit of discovery.

Translation feature hypotheses explicitation and can be called clarity, of explicit, explicit, express, etc. "refers to a tendency to express textual information may be implied." Manifest hypothesis was first proposed by the Blum-Kulka, she based translator clear process of convergence means translation in the translation process, so that the relationship between Mr Cohesion more clearly put forward the hypothesis. Blum-Kulka believes that the translator needs to be successful translation of discourse and discourse complicated process, may lead to increased target text than the length of the source text, so that the relationship between cohesion translation clearer than the original.

Before manifest assumptions, scholars have been significant phenomenon discussed. As Vanderawera pointed out the various manifestation / clarity: using parenthesis indicate more clearly thought process of a particular character or emphasize understanding; extended chapter concentrates; add the qualifier qualifiers and conjunctions to improve transparency; add extra information; add interpreted language; a clearer expression and repeat the previous details; clearly implied or ambiguous information; more precise description, location clear, with a clear identity within the meaning of definite pronouns like.

About manifest motives, domestic and foreign scholars have discussed. Blmn-Kulka believes that the translation process is the obvious inherent properties and Translator style related, but has nothing to do with the differences between the source and target language of the language and culture. Wang Ke non-research shows that in Translation emerged Translated Text amplification characteristics, which can manifest as a parameter; at the same time, interference and migration primitives is a factor to be reckoned with. Pym from the principles of cooperation and risk management perspective on the significant Motives explained: First, in order to achieve cooperation with the target readers, that smooth communication, the translator will understand as much as possible to provide the translation communicative clues to target readers; Second, in order to avoid the risk of the translator and the use of manifest translation strategy, because this strategy reduced the risk of translation. Ke Fei believes that translation as a phenomenon, manifest should not just narrowly refers to the convergence of language change in form, it should also include the conversion manifest sense, that facilitates translation add translation readers manifest expression of understanding, or that the original information was implicit in the translation technology to make it more specific, clearer logic. He also noted that the translation is explicit and implicit coexistence, implicit, explicit and extent of the phenomenon may be related to the degree of formal language and translation direction about the same time translator factors, text factors, language differences and cultural differences also lead to significant phenomenon factors produced. Wang Lirong explains significant Motives based on cognitive framework that different languages and cultures of the recipient of cognitive differences and significant phenomenon of the same coin has a direct relationship. Hu Bao will manifest motivation summarized as follows: the translation process motivation, language and cultural differences motivation translator.

Hidden features of translation is the implicit and explicit concepts of opposed, Huang Libo think Invisiblization means "faded process description language component according to Target Language Specification." Hu Bao interpreted as: "In the process of translation, the translator, without prejudice to the reader in understanding, in order to make the target text concise, focused, avoid words expressing tired Chan, bloated and often clear the source language text syntactic or semantic relationship expressed without hidden information table."

Translation of feature assumptions explicit / implicit hypothesis of our research is directly related. Our findings can be used to manifest / implicit assumption of the theory of interpretation and we can also validate these theoretical assumptions or improvements. Although the rise of Corpus Translation Studies and applications related to research methods, greatly enriched the translation feature assumptions manifest / hidden and so the scope of the study, but the translation is assumed characteristics still exist some problems. 


\section{The Real Effect on the Translation Based on English Corpus}

Corpus-based Translation Studies is this growing area of research methods to promote the translation from the Statute of the steering descriptive. It makes the initial translation studies from the perspective of linguistics and comparative linguistics point of view, developed later to interpret the translations from multiple perspectives of social, political, cultural and other internal and external characteristics, which marked its development trend from mere description to describe the study, Interpretation of Research and development.

However, we should clearly recognize that, just like the use of TEC with comparable Translation Corpus Translation Studies now it seems there are still some limitations, some scholars have suggested that combined comparable corpus and parallel corpus to translate research system?. Although this is theoretically possible, but so far, the world has not yet been completed and that the system design, the combination of these two characteristics multifunction large translation corpus, therefore, to be further strengthened in this respect the basic theory the study.

In any case, the positive comments of Corpus-based Translation Studies, one thing is self-evident that it is by means of modern computer technology, not only from the perspective of linguistics and comparative linguistics, but also from the political, ideological, economic , a macro point of culture to study translation phenomena reveal translation exists probable of law, systematically summarizes the translated text and translation process of "total translation feature" which translation has been a comprehensive understanding of the wide-angle, which in even with previous investigators personal intuition and experience is difficult to achieve, thus ample evidence of the role and significance of Corpus - based translation studies. As Tymoczko said: CTS is a powerful tool that not only allows us to fully understand the differences arising from translation of conversion, and this summed up the difference between the law can play a role in the formation of norms.

\section{Conclusion}

The translation study based on the corpus is unique, since it was built, many scholars use it made a lot of achievement and it let the world with admiration. At the same time, people have also built other languages translation corpus imitating translated corpus, established a new research paradigm as an important transformation in translation studies, so that this new type of translation studies showing booming situation and established an important position in the world of translation studies.

\section{Acknowledgements}

Fund Project: Humanities and Social Science Fund Project: the English Parallel Corpus Construction of Cultural Words in Grassland Culture (NJSY16009)

\section{References}

[1] Jia Xinzhang, Li Jingyuan. Foreign Language Study, Vol. 6 (2004) No 53, p.25-26

[2] Peng Sue, Wang Yunhui, Wang Qunyong. Shanghai Translation, Vol. 12 (2005) No 27, p.74-76

[3] Qian Xiyuan, Jing Jianfen, Hou XuSiem. East China Normal University, Vol. 30 (2004) No 19, p.144-145

[4] Wang Kuailiang. Foreign Language Teaching and Research, Vol. 29 (2008) No 27, p.21-23

[5] Zhang Gongxu, Sun Jing. Chinese Translation, Vol. 8 (2003) No 27, p.57-60 